\title{
Erratum to: 7TM Domain Structure of Adhesion GPCRs
}

\section{Saskia Nijmeijer, Steffen Wolf, Oliver P. Ernst, and Chris de Graaf}

(C) Springer International Publishing AG 2016

T. Langenhan, T. Schöneberg (eds.), Adhesion G Protein-coupled Receptors, Handbook of Experimental Pharmacology 234,

DOI 10.1007/978-3-319-41523-9_3

In the chapter titled "7TM Domain Structure of Adhesion GPCRs", the sequence of the author name was incorrect. The correct sequence should read as follows:

Saskia Nijmeijer, Steffen Wolf, Oliver P. Ernst, and Chris de Graaf

The updated original online version for this chapter can be found at

DOI 10.1007/978-3-319-41523-9_3

\footnotetext{
S. Nijmeijer • C. de Graaf $(\bowtie)$

Department of Medicinal Chemistry, Amsterdam Institute for Molecules, Medicines and Systems, Vrije Universiteit Amsterdam, Amsterdam 1081HV, The Netherlands e-mail: s.nijmeijer@vu.nl; c.de.graaf@vu.nl

S. Wolf

Department of Biophysics, CAS-MPG Partner Institute for Computational Biology, Key Laboratory of Computational Biology, Shanghai Institutes for Biological Sciences, Chinese Academy of Sciences, Shanghai, P.R. China

Institute of Physics, Albert Ludwigs University, Freiburg, Germany

e-mail: swolf@picb.ac.cn

O.P. Ernst

Department of Biochemistry, University of Toronto, 1 King's College Circle, Toronto, ON, Canada, M5S 1A8

Department of Molecular Genetics, University of Toronto, 1 King's College Circle, Toronto, ON, Canada, M5S 1A8

e-mail: oliver.ernst@utoronto.ca
} 